\title{
Parallel Reaction Monitoring
}

National Cancer Institute

\section{Source}

National Cancer Institute. Parallel Reaction Monitoring. NCI Thesaurus. Code C161788.

A method for collecting tandem mass spectrometry data where an ion of a particular mass is selected in the first stage and a fragmented ion product is selected in the second mass spectrometer stage for detection. Parallel reaction monitoring (PRM)-based experiments are generally performed in a high-resolution hybrid quadrupole-Orbitrap (QOT) or time-of-flight mass spectrometer. 